九州大学学術情報リポジトリ

Kyushu University Institutional Repository

\title{
A RANDOM WALK AND ITS LIL IN A BANACH SPACE
}

Chang, Mou-Hsiung

Department of Mathematics, The University of Alabama in Huntsville

https://doi.org/10.5109/13125

出版情報: 統計数理研究. 18 (1/2)，pp.81-91，1978-03. Research Association of Statistical Sciences

バージョン：

権利関係 : 


\title{
A RANDOM WALK AND ITS LIL IN A BANACH SPACE*
}

\author{
By
}

\section{Mou-Hsiung CHANG**}

(Received October 8, 1977)

\begin{abstract}
Let $\left\{X_{n}: n \geqq 1\right\}$ be a sequence of i.i.d. Banach space valued random variables with $E\left[X_{n}\right]=0$ and $E\left\|X_{n}\right\|^{2}<\infty$, and let $S_{0}=0$, $S_{n}=X_{1}+X_{2}+\ldots+X_{n}, n \geqq 1$. We prove that if $\left\{S_{n}: n \geqq 1\right\}$ satisfies the LIL in $B$ then the sequence $\left\{\eta_{n}: n \geqq 1\right\}$ satisfies the LIL in $C([0,1], B)$, where $\eta_{n}(t)=S_{[n t]}+(n t-[n t]) \quad X_{[n t]+1}, \quad 0 \leqq t \leqq 1$ and $C([0,1], B)=\{f:[0,1] \rightarrow B \mid f$ is continuous $\}$. We also use this result to give an alternative to the proof of the LIL of Brownian motion in Banach spaces.
\end{abstract}

\section{Introduction}

Let $B$ be a real separable Banach space with the norm $\|\cdot\|$ and $B^{*}$ be its topological dual. Throughout, $\left\{X_{n}: n \geqq 1\right\}$ always denotes a sequence of i. i.d. $B$-valued random variables on a probability space $(\Omega, \mathcal{A}, P)$ with $E\left(X_{n}\right)=0$ and $E\left\|X_{n}\right\|^{2}<\infty$. Note that $E\left\|X_{n}\right\|^{2}<\infty$ assures the existence of a covariance operator

$$
T(f, g)=E\left[f\left(X_{n}\right) g\left(X_{n}\right)\right], \quad f, g \in B^{*} .
$$

Let $\mu$ denote the mean zero Gaussian measure on $B$ with the given covariance operator whenever this measure exists. Let $H \mu \subseteq B$ denote the reproducing kernel Hilbert space of $\mu$. This pair of spaces $(B, H \mu)$ is often referred to as an abstract Wiener space [4]. Perhaps one of the most important properties of abstract Wiener space is the existence of a constant $M>0$ such that $\|x\| \leqq M\|x\|_{\mu}$ for every $x$ in $H_{\mu}$, where $\|\cdot\|_{\mu}$ is the norm of $H_{\mu}$. Consequently, through the continuous injection $i: H_{\mu} \rightarrow B$ and the restriction map $i^{*}: B^{*} \rightarrow H_{\mu}^{*}$ we have the relation $B^{*} \subseteq H_{\mu}^{*} \approx H_{\mu} \subseteq B$. Let $\{W(t)$ : $t \geqq 0\}$ denote $\mu$-Brownian motion with the transition probability $P_{t}(a, A)=\mu\left((A-a) / t^{1 / 2}\right)$. It is known that $\{W(t): 0 \leqq t \leqq 1\}$ induces a mean zero, Gaussian measure $P_{w}$ on the

* Supported by a grant from Research Grants Committee of the University of Alabama in Huntsville.

** Department of Mathematics, The University of Alabama in Huntsville, Huntsville, Alabama 35807, USA. 
measure space $\left(C_{B}, \mathscr{F}\right)$, where $C_{B}$ is the space of continuous functions $w$ from $[0,1]$ into $B$ with $w(0)=0$, and $\mathscr{I}$ is the $\sigma$-field generated by the functions $w \rightarrow w(t) . P_{w}$ is called abstract Wiener measure. See [4], [5] and [13] for expositions of concepts of $\mu$-Brownian motion.

In this paper, we are interested in the random walk $\left\{\eta_{n}: n \geqq 1\right\}$ defined by

$$
\eta_{n}(t)=S_{-n t]}+(n t-[n t]) X_{[n t]+1}, \quad 0 \leqq t \leqq 1,
$$

where $S_{0}=0$,

$$
S_{k}=X_{1}+X_{2}+\cdots+X_{k}
$$

for $k \geqq 1$ and $[r]$ mean the greatest integer which is less than or equal to $r$. We say that the sequence $\left\{X_{n}: n \geqq 1\right\}$ satisfies the central limit theorem (CLT) in $B$ if the distribution of $S_{n} / n^{1 / 2}$ converges weakly to $\mu\left(\mathcal{L}\left(S_{n} / n^{1 / 2}\right) \Rightarrow \mu\right)$. We say that the sequence $\left\{S_{n}: n \geqq 1\right\}$ satisfies the law of iterated logarithm (LIL) in $B$ if there exists a compact, symmetric convex $K \subset B$ such that

$$
P\left\{\lim _{n} d\left(S_{n} /(2 n L L n)^{1 / 2}, K\right)=0\right\}=1
$$

and

$$
P\left\{C\left(\left\{S_{n} /(2 n L L n)^{1 / 2}\right\}\right)=K\right\}=1,
$$

where

$$
d(x, K)=\inf _{y \in K}\|x-y\|,
$$

$C\left(\left\{X_{n}\right\}\right)$ means the set of strong limit points of the sequence $\left\{X_{n}: n \geqq 1\right\}$ in $B$ and $L L n=1$ if $n=1,2,=\log \log n$ if $n \geqq 3$. The equivalence among the boundedness of $E\left\|X_{n}\right\|^{2}$, CLT and LIL are well known in [6], [15] and [16] when $B=\boldsymbol{R}^{k}$. However, when $B$ is a general Banach space, there is no implication among those three concepts as can be seen in [2], [7] and [10]. The main purpose of this paper is to show that the LIL of $\left\{S_{n}: n \geqq 1\right\}$ in $B$ implies the LIL of $\left\{\eta_{n}: n \geqq 1\right\}$ in $C_{B}$. We also use this result to give an alternative to the proof of the LIL of Brownian motion in Banach spaces. A work of the same spirit but different content is [8] in which $\mathcal{L}\left(S_{n} / n^{1 / 2}\right) \Rightarrow \mu$ implies $\mathcal{L}\left(\eta_{n} / n^{1 / 2}\right) \Rightarrow P_{w}$ has been established.

The following necessary and sufficient condition for LIL in $B$ will be used in proving our main result.

Theorem 1. (Kuelbs $\left[11\right.$, p. 745]) Let $X_{1}, X_{2}, \cdots$ be i.i.d. B-valued such that $E\left(X_{n}\right)=0$ and $E\left\|X_{n}\right\|^{2}<\infty$. Then the sequence $\left\{S_{n}: n \geqq 1\right\}$ satisfies the LIL in $B$ if and only if

$$
P\left\{\left\{S_{n} /(2 n L L n)^{1 / 2}: n \geqq 1\right\} \text { is relatively compact in } B\right\}=1 \text {. }
$$

For other results on LIL in abstract spaces, see [9], [12], [13] and [14]. 


\section{Main result}

Let $C([0,1], B)$ be the space of continuous functions $f$ from $[0,1]$ into $B$. $C([0,1], B)$ is a real separable Banach space under the norm

$$
\|f\|_{\infty}=\sup _{0 \leq t \leqq 1}\|f(t)\| \text {. }
$$

A family

$$
\left\{f_{\alpha}: \alpha \in A\right\} \subset C([0,1], B)
$$

is said to be uniformly equicontinuous if

$$
\lim _{\delta \rightarrow 0} \sup _{\alpha \in A} \sup _{\mid s-t<\delta}\left\|f_{\alpha}(s)-f_{\alpha}(t)\right\|=0
$$

The following lemma (without condition (ii)) is known as the Arzelá-Ascoli theorem when $\operatorname{dim}(B)<\infty$. However in a general Banach space $B$, its proof does not seem to be available in literature. Therefore, we include its proof in the Appendix for the sake of completeness.

Lemma 1. (Arzelá-Ascoli Theorem) A subset $\left\{f_{\alpha}: \alpha \in A\right\}$ is relatively compact in $C([0,1], B)$ if and only if

(i) $\sup _{\alpha \in \boldsymbol{A}}\left\|f_{\alpha}(0)\right\|<\infty$,

(ii) for each $t$ in $[0,1]$, the set $\left\{f_{\alpha}(t): \alpha \in A\right\}$ is relatively compact in $B$ and

(iii) $\left\{f_{\alpha}: \alpha \in A\right\}$ is uniformly equicontinuous in $C([0,1], B)$.

Theorem 2. (Main Result) Let $\left\{X_{n}: n \geqq 1\right\}$ be a sequence of i.i.d. B-valued random variables such that $E\left(X_{n}\right)=0$ and $E\left\|X_{n}\right\|^{2}<\infty$, and let $\left\{\eta_{n}: n \geqq 1\right\}$ be as in (1). Then $\left\{S_{n}: n \geqq 1\right\}$ satisfies the LIL in $B$ if and only if $\left\{\eta_{n}: n \geqq 1\right\}$ satisfies the LIL in $C_{B}$.

Proof. The sufficiency of the theorem is trivial. Therefore we shall only prove the necessity. By Lemma 1 and Theorem 1, this is equivalent to show that the following three conditions hold.

(i) $P\left\{\left\{\eta_{n}(0) / 2 n L L n\right)^{1 / 2}: n \geqq 1\right\}$ is bounded in $\left.B\right\}=1$;

(ii) $P\left\{\left\{\eta_{n}(t) / 2 n L L n\right)^{1 / 2}: n \geqq 1\right\}$ is relatively compact in $\left.B\right\}=1$ for each $t$ in $[0,1]$; and

(iii) $P\left\{\left\{\eta_{n} /(2 n L L n)^{1 / 2}: n \geqq 1\right\}\right.$ is uniformly equicontinuous in $\left.C_{B}\right\}=1$.

Condition (i) follows immediately from the fact that for $n=1,2, \cdots \quad P\left\{\eta_{n}(0)=0\right\}=1$. The validity of (ii) and (iii) are treated in Lemma 2 and Lemma 3 respectively.

Lemma 2. Let $\left\{X_{n}: n \geqq 1\right\}$ and $\left\{\eta_{n}: n \geqq 1\right\}$ be as in Theorem 2. If $\left\{S_{n}: n \geqq 1\right\}$ satisfies the LIL in $B$ then for each $t$ in $[0,1]$

(6) $P\left\{\left\{\eta_{n}(t) /(2 n L L n)^{1 / 2}: n \geqq 1\right\}\right.$ is relatively compact in $\left.B\right\}=1$. 
Proof. By Theorem 1, there exists a compact, symmetric, convex $K \subset B$ such that $P\left(\Omega_{1}\right)=P\left(\Omega_{2}\right)=1$, where

$$
\Omega_{1}=\left\{\lim _{n} d\left(S_{n} /(2 n L L n)^{1 / 2}, K\right)=0\right\}
$$

and

$$
\Omega_{2}=\left\{C\left(\left\{S_{n} /(2 n L L n)^{1 / 2}\right\}\right)=K\right\} .
$$

Now let $\omega \in \Omega_{1} \cap \Omega_{2}$ and $t \in[0,1]$ be given (when $t=0$ the conclusion is trivial), and let $\tilde{K}=a_{t} K=\left\{a_{\imath} y: y \in K\right\}$, where

$$
a_{t}=\lim _{n}([n t] L L[n t] / n L L n)^{1 / 2} .
$$

We claim that

$$
\lim _{n} d\left(\eta_{n}(t, \omega) /(2 n L L n)^{1 / 2}, \tilde{K}\right)=0
$$

and

$$
C\left(\left\{\eta_{n}(t, \omega) /(2 n L L n)^{1 / 2}\right\}\right)=\tilde{K} .
$$

These two conclusions will then imply (6), by Theorem 1 .

For the proof of (7), we simply observe that

$$
\lim _{n}\left\|X_{[n t]+1}(\omega) /(2 n L L n)^{1 / 2}\right\|=0 .
$$

Then

$$
\begin{aligned}
& \lim _{n} \inf _{y \in K}\left\|\eta_{n}(t, \omega) /(2 n L L n)^{1 / 2}-z\right\| \\
& \quad \leqq \lim _{n} \inf _{y \in K} a_{t}\left\|S_{[n t]}(\omega) /(2[n t] L L[n t])^{1 / 2}-y\right\| \\
& \quad+\lim _{n}\left\|X_{[n t]+1}(\omega) /(2 n L L n)^{1 / 2}\right\|=0 .
\end{aligned}
$$

For the proof of (8), let $z \in \tilde{K}$. Then $z=a_{t} y$ for some $y \in K$. By the assumption, we have a subsequence $\{n(k): k \geqq 1\}$ of $\{n: n \geqq 1\}$ such that

$$
\lim _{k}\left\|S_{n(k)}(\omega) /(2 n(k) L L n(k))^{1 / 2}-y\right\|=0 .
$$

This implies that

$$
\lim _{k}\left\|\eta_{n(k)}(t) /(2 n(k) \operatorname{LLn}(k))^{1 / 2}-z\right\|=0
$$

by the same argument as in the proof of (7).

REMARK 1 . The proof of Lemma 2 actually yields that if $\left\{S_{n}: n \geqq 1\right\}$ satisfies LIL in $B$ then

(9) $P\left\{\left\{\left(\eta_{n}(t)-\eta_{n}(s)\right) / \lambda(n, t, s): n \geqq 1\right\}\right.$ is relatively compact in $\left.B\right\}=1$ for any $s, t$ in $[0,1]$ with $s<t$, where

$$
\lambda(n, t, s)=\{2([n t]-[n s]) L L([n t]-[n s])\}^{1 / 2} .
$$

This remark will be used in the proof of Lemma 3. 
Lemma 3. Let $\left\{X_{n}: n \geqq 1\right\}$ and $\left\{\eta_{n}: n \geqq 1\right\}$ be as in Theorem 2. If $\left\{S_{n}: n \geqq 1\right\}$ satisfies the LIL in $B$ then

(10) $P\left\{\left\{\eta_{n} /(2 n L L n)^{1 / 2}: n \geqq 1\right\}\right.$ is uniformly equicontinuous in $\left.C_{B}\right\}=1$.

PROOF. Let $\beta>1$ be fixed, and let $n_{r}=\left[\beta^{r}\right]$. We shall prove that

(11) $P\left\{\left\{\eta_{n_{r}} /\left(2 n_{r} L L n_{r}\right)^{1 / 2}: r \geqq 1\right\}\right.$ is uniformly equicontinuous in $\left.C_{B}\right\}=1$.

Let $\Gamma>0$ be such that for $n \geqq 1$ and $s, t \in[0,1]$

$$
P\left\{\left\|\eta_{n}(t)-\eta_{n}(s)\right\|>\Gamma \lambda(n, t, s)\right\}=0 .
$$

This $\Gamma$ exists by Remark 1. For $r=1,2, \cdots$ and $m_{0}$ an arbitrary positive integer, define $A_{r}$ by

$$
A_{r}=\bigcup_{m=m 0}^{\infty} \bigcup_{k=1}^{2 m} A_{r k m}
$$

where

$$
A_{r k m}=\left\{\left\|\eta_{n_{r}}\left(k 2^{-m}\right)-\eta_{n_{r}}\left((k-1) 2^{-m}\right)\right\|>\varepsilon_{m}\left(2 n_{r} L L n_{r}\right)^{1 / 2}\right\}
$$

and $\varepsilon_{m}=2^{-m / 2} \Gamma$. It is clear that (11) holds if $P\left\{A_{r}\right.$ i.o. in $\left.r\right\}=0$ since $\varepsilon_{m} \downarrow 0$ as $m \rightarrow \infty$. Now

$$
P\left(A_{r k m}\right)=P\left\{\left\|\eta_{n_{r}}\left(k 2^{-m}\right)-\eta_{n_{r}}\left((k-1) 2^{-m}\right)\right\| / \lambda\left(n_{r}, k 2^{-m},(k-1) 2^{-m}\right) \geqq \varepsilon_{m} \theta(r, m, k)\right\}
$$

where

$$
\theta(r, m, k)=\left(2 n_{r} L L n_{r}\right)^{1 / 2} / \lambda\left(n_{r}, k 2^{-m},(k-1) 2^{-m}\right)
$$

and $\lambda(n, t, s)$ is as in (9). We choose $m_{0}$ sufficiently large such for $m \geqq m_{0}$

Then

$$
\lambda\left(n_{r}, k 2^{-m},(k-1) 2^{-m}\right) \leqq 2^{-m / 2}\left\{2 n_{r} L L n_{r}\right\}^{1 / 2} .
$$

$$
\begin{aligned}
P\left(A_{r}\right) & \leqq \sum_{m=m_{0}}^{\infty} \sum_{k=1}^{2 m} P\left(A_{r k m}\right) \\
& \leqq \sum_{m=m_{0}}^{\infty} \sum_{k=1}^{2 m} P\left\{\left\|\eta_{n_{r}}\left(k 2^{-m}\right)-\eta_{n_{r}}\left((k-1) 2^{-m}\right)\right\| / \lambda\left(n_{r}, k 2^{-m},(k-1) 2^{-m}\right) \varepsilon^{m} 2^{-m / 2}\right\} \\
& =\sum_{m=m_{0}}^{\infty} \sum_{k=1}^{2^{m}} P\left\{\left\|\eta_{n_{r}}\left(k 2^{-m}\right)-\eta_{n_{r}}\left((k-1) 2^{-m}\right)\right\|>\Gamma \lambda\left(n_{r}, k 2^{-m},(k-1) 2^{-m}\right)\right\} \\
& =0 \text { by Remark } 1 \text { for } \quad r=1,2, \cdots .
\end{aligned}
$$

Consequently

$$
\sum_{r=1}^{\infty} P\left(A_{r}\right)=0
$$

Thus by Borel-Cantelli's lemma, we have proved $P\left\{A_{r}\right.$ i. o. in $\left.r\right\}=0$.

We next want to prove that for each $\varepsilon>0$ there exists a fixed $\beta_{0}>1$ such that for all $\beta$ satisfying $1<\beta \leqq \beta_{0}$

$$
P\left\{C_{r} \text { i. o. in } r\right\}=0 \text {, }
$$


where

$$
C_{r}=\left\{\max _{n_{r-1} \leq k \leq n_{r}}\left\|\eta_{n_{r-1}} /\left(2 n_{r-1} L L n_{r-1}\right)^{1 / 2}-\eta_{k} /(2 k L L k)^{1 / 2}\right\|_{\infty}>\varepsilon\right\}
$$

This together with (11) will then conclude the lemma. Define

$$
D_{r}=\left\{\max _{n_{r-1} \leq k \leq n_{r}}\left\|\eta_{n_{r-1}}-\eta_{k}\right\|_{\infty}>\varepsilon\left(2 n_{r-1} L L n_{r-1}\right)^{1 / 2}\right\}
$$

and

$$
E_{r}=\left\{\max _{n_{r-1} \leq k \leqq n_{r}} \| \eta_{k}\left((2 k L L k)^{-1 / 2}-\left(2 n_{r-1} L L n_{r-1}\right)^{-1 / 2} \|_{\infty}>\varepsilon / 2\right\} .\right.
$$

Let $\alpha=\varepsilon /\left[4\left(\beta^{1 / 2}-1\right)\right]$. We have

$$
\begin{aligned}
& P\left(E_{r}\right) \leqq P\left\{\max _{k \leqq n_{r}}\left\|\eta_{k}\right\|_{\infty}>\alpha\left(2 n_{r} L L n_{r}\right)^{1 / 2}\right\} \\
& \leqq \sum_{k=1}^{n_{r}} P\left\{\left\|\eta_{k}\right\|_{\infty}>\alpha(2 k L L k)^{1 / 2}\right\} \\
& \leqq \sum_{k=1}^{n_{r}} P\left\{\sup _{0<|s-t| \leqq 1}\left\|\eta_{k}(t)-\eta_{k}(s)\right\|>\alpha(2 k L L k)^{1 / 2}\right\} \\
& \leqq \sum_{k=1}^{n_{r}} P\left\{\sup _{0<|s-t| \leq 2^{-} m}\left\|\eta_{k}(t)-\eta_{k}(s)\right\|>\alpha(2 k L L k)^{1 / 2} / 2^{m}\right\} \\
& \text { for any } m \geqq 1 .
\end{aligned}
$$

Let $\beta>1$ be sufficiently close to one such that

$$
\alpha / 2^{m}=\varepsilon / 2^{m+2}\left(\beta^{1 / 2}-1\right)>\Gamma,
$$

where $\Gamma$ is as in (12). Then

(14)

$$
\begin{aligned}
& P\left(E_{r}\right) \\
& \quad \leqq \sum_{k=1}^{n_{r}} P\left\{\sup _{0<\mid s-t<2^{-m}}\left\|\eta_{k}(t)-\eta_{k}(s)\right\|>\Gamma(2 k L L k)^{1 / 2}\right\} \\
& \leqq \sum_{k=1}^{n_{r}} \sum_{j=1}^{2^{m}} P\left\{\left\|\eta_{k}\left(j 2^{-m}\right)-\eta_{k}\left((j-1) 2^{-m}\right)\right\|>\Gamma(2 k L L k)^{1 / 2}\right\} \\
& =0 \text { by Remark } 1 .
\end{aligned}
$$

Now

$$
\begin{aligned}
& P\left(D_{r}\right) \\
& \leqq \sum_{k=n_{r-1}}^{n_{r}} \sum_{j=1}^{k-n} \sum_{r-1}^{1^{+1}} P\left\{\left\|Y_{r j}\right\|_{\infty}>\gamma(r, j)\right\} \\
& \leqq \sum_{k=n_{r-1}}^{n_{r}} \sum_{j=1}^{k-n_{r-1} k^{+1}} P\left\{\sup _{0<\mid s-t \leq 2^{-m}}\left\|Y_{r j}(t)-Y_{r j}(s)\right\|>\gamma(r, j) / 2^{m}\right\},
\end{aligned}
$$


where

$$
Y_{r j}=\eta_{n_{r-1}+j}-\eta_{n_{r-1}+j-1}
$$

and

$$
\gamma(r, j)=\left(2\left(n_{r}-n_{r-1}\right) L L\left(n_{r}-n_{r-1}\right)\right)^{1 / 2} / 4 j(\beta-1)^{1 / 2} .
$$

Choosing $\beta>1$ sufficiently close to 1 so that

$$
\varepsilon / j 2^{m+2}(\beta-1)^{1 / 2}>\Gamma,
$$

where $\Gamma$ is as in (12), and applying Remark 1 and argument as in (14), we have

$$
\begin{aligned}
& P\left\{\sup _{0<1 s-t<2^{-} m}\left\|Y_{r j}(t)-Y_{r j}(s)\right\|>\gamma(r, j) / 2^{m}\right\} \\
& \leqq P\left\{\sup _{0<|s-t| \leqq 2^{-} m}\left\|Y_{r j}(t)-Y_{r j}(s)\right\|>\gamma(r, j) / 2^{m}\right\} \\
& \leqq P\left\{\sup _{0<\mid s-t \leq 2^{-m}}\left\|Y_{r j}(t)-Y_{r j}(s)\right\|>\phi(r)\right\} \\
& \leqq \sum_{l=1}^{2 m} P\left\{\left\|\eta_{n_{r-1^{+}}}\left(l 2^{-m}\right)-\eta_{n_{r^{-1}+j}}\left((l-1) 2^{-m}\right)\right\|>\phi(r) / 2\right\} \\
& \leqq \sum_{l=1}^{2 m} P\left\{\left\|\eta_{n_{r-1}+j-1}\left(l 2^{-m}\right)-\eta_{n_{r-1}+j-1}\left((l-1) 2^{-m}\right)\right\|>\phi(r) / 2\right\} \\
& =0 \text {, }
\end{aligned}
$$

where

$$
\phi(r)=\Gamma\left(2\left(n_{r}-n_{r-1}\right) L L\left(n_{r}-n_{r-1}\right)\right)^{1 / 2} .
$$

Thus $P\left(D_{r}\right)=0$. Now

$$
\sum_{r=1}^{\infty} P\left(C_{r}\right) \leqq \sum_{r=1}^{\infty} P\left(D_{r}\right)+\sum_{r=1}^{\infty} P\left(E_{r}\right)=0
$$

By the Borel-Cantelli lemma, we have $P\left(C_{r}\right.$ i. o. in $\left.r\right)=0$. This completes the proof of Lemma 3.

\section{An application}

Let $\left\{Z_{n}: n \geqq 1\right\}$ be a sequence of independent copies of $Z$, where $Z$ is the mean zero, $B$-valued Gaussian random variable whose distribution is $\mu$. We define

$$
W_{n}(t)=\sum_{i=0}^{[n t]} Z_{i}+(n t-[n t]) Z_{[n t]+1}, 0 \leqq t \leqq 1 .
$$

Note that for each $n=1,2, \cdots$ the stochastic process $\left\{W_{n}(t): 0 \leqq t \leqq 1\right\}$ is essentially the polygonalized Brownian motion. That is

$$
W_{n}\left(\frac{l}{n}\right)=W(l)
$$


for $l=1,2, \cdots, n$, and is linear on intervals $[(l-1) / n, l / n]$. It is known from $[14]$ that

$$
\left\{\sum_{i=1}^{n} Z_{i}: n \geqq 1\right\}
$$

satisfies the LIL in $B$. Therefore the sequence $\left\{W_{n}: n \geqq 1\right\}$ satisfies the LIL in $C_{B}$ by Theorem 2. In this section, we shall illustrate an alternative to the proof of the LIL for Brownian motion in Banach space given by Kuelbs and LePage [13].

Theorem 3. (Kuelbs and LePage [13]) Let $\{W(t): t \geqq 0\}$ be $\mu$-Brownian motion in $B$. Then the sequence $\left\{\xi_{n}: n \geqq 1\right\}$ satisfies the $L I L$ in $C_{B}$, where $\xi_{n}(t)=W(n t)$, $0 \leqq t \leqq 1$. Furthermore the compact, symmetric convex $K \subset C_{B}$ described in (2) and (3) can be characterized as follows:

$$
\begin{aligned}
& K=\left\{f \in C_{B}: f(t) \in H_{\mu} \text { for each } t \in[0,1],\right. \text { and } \\
&\left.\sum_{j} \int_{0}^{1}\left[(d / d t) x_{j}^{*}(f)(t)\right]^{2} d t \leqq 1\right\},
\end{aligned}
$$

where $\left\{x_{j}^{*}: j \geqq 1\right\} \subset B^{*}$ is such that the set

$$
\left\{\int_{B} x_{j}^{*}(x) x d \mu(x): j \geqq 1\right\}
$$

forms a complete orthonormal system for $H_{\mu}$.

Proof. The characterization of the set $K$ in (16) is from Lemma 4 of [13]. It remains to show that

$$
P\left\{\lim _{n}\left\|\xi_{n}-W_{n}\right\|_{\infty} /(2 n L L n)^{1 / 2}=0\right\}=1 .
$$

This together with the fact that $\left\{W_{n}: n \geqq 1\right\}$ satisfies the LIL in $C_{B}$ will then imply that $\left\{\xi_{n}: n \geqq 1\right\}$ satisfies the LIL in $C_{B}$.

Let $\left\{\varepsilon_{n}: n \geqq 1\right\}$ be a sequence of positive real numbers whose precise values will be determined later. We have

$$
\begin{aligned}
P\left\{\| \xi_{n}\right. & \left.-W_{n} \|_{\infty} /(2 n L L n)^{1 / 2} \geqq \varepsilon_{n}\right\} \\
& \leqq \sum_{k=0}^{n-1} P\left\{\sup _{k / n \leq t \leq(k+1) / n}\left\|W(n t)-W_{n}(t)\right\| \geqq \varepsilon_{n}(2 n L L n)^{1 / 2}\right\} \\
& =\sum_{k=0}^{n-1} P\left\{\sup _{0 \leq t \leq n-1}\|W(n t)-n t W(1)\| \geqq \varepsilon_{n}(2 n L L n)^{1 / 2}\right\}
\end{aligned}
$$

since

$$
W_{n}\left(\frac{l}{n}\right)=W(l)
$$

and is linear on $[l / n,(l+1) / n]$ for $l=0,1,2, \cdots, n ; n=1,2, \cdots$. 
Now

$$
\begin{aligned}
& P\left\{\sup _{0 \leqq t \leqq n^{-1}}\|W(n t)-n t W(1)\| \geqq \varepsilon_{n}(2 n L L n)^{1 / 2}\right\} \\
& \quad \leqq P\left\{\sup _{0 \leqq t \leqq n^{-1}}\|W(n t)-n t W(n t)\| \geqq \varepsilon_{n}(2 n L L n)^{1 / 2} / 2\right\} \\
& \quad+P\left\{\sup _{0 \leqq t \leqq n^{-1}}\|n t W(n t)-n t W(1)\| \geqq \varepsilon_{n}(2 n L L n)^{1 / 2} / 2\right\} \\
& \quad \leqq 5 P\left\{\|W(1)\| \geqq \varepsilon_{n}(2 n L L n)^{1 / 2} / 4\right\}
\end{aligned}
$$

From Fernique's estimate [2], there exist constants $\gamma>0$ and $C>0$ such that

$$
\exp \left\{r\|W(1)\|^{2}\right\} \leqq C .
$$

Applying Chevyshev's inequality and Fernique's estimate to the last expression in (17), we have

$$
\begin{aligned}
& P\left\{\sup _{0 \leqq t \leqq n^{-1}}\|W(n t)-n t W(1)\| \geqq \varepsilon_{n}(2 n L L n)^{1 / 2}\right\} \\
& \quad \leqq 5 C \exp \left\{-\gamma \varepsilon_{n}{ }^{2} n L L n / 8\right\} \text {. Choose } \varepsilon_{n}=(L L n)^{-1 / 2} .
\end{aligned}
$$

Then $\varepsilon_{n} \downarrow 0$ as $n \rightarrow \infty$ and we have

$$
\begin{gathered}
\sum_{n=1}^{\infty} P\left\{\left\|\xi_{n}-W_{n}\right\|_{\infty} /(2 n L L n)^{1 / 2} \geqq \varepsilon_{n}\right\} \\
\leqq \sum_{n=1}^{\infty} 5 C n \exp \{-\gamma n / 8\}<\infty
\end{gathered}
$$

By Borel-Cantelli's lemma, this implies that

$$
P\left\{\lim _{n}\left\|\xi_{n}-W_{n}\right\|_{\infty} /(2 n L L n)^{1 / 2}=0\right\}=1 .
$$

\section{Appendix: Proof of Lemma 1}

The necessity of Lemma 1 follows exactly as that of the Arzelá-Ascoli theorem in $C[0,1]$ (see e.g. $[1$, p. 221]). We only have to prove the sufficiency.

Now assume that conditions (i)-(iii) hold. Let $\varepsilon>0$ be given. Choose $k$ large enough that

$$
\sup _{\alpha \in A} \sup _{|s-t|<1 / k}\left\|f_{\alpha}(s)-f_{\alpha}(t)\right\|<\varepsilon .
$$

Since

$$
\left\|f_{\alpha}(t)\right\| \leqq\left\|f_{\alpha}(0)\right\|+\sum_{i=1}^{k}\left\|f_{\alpha}(i t / k)-f_{\alpha}((i-1) t / k)\right\|,
$$

it follows that

$$
\sup _{0 \leq t \leq 1} \sup _{\alpha \in A}\left\|f_{\alpha}(t)\right\| \equiv C<\infty . \quad \text { Let } \quad K=\bigcup_{i=0}^{k}\left\{f_{\alpha}(i / k): \alpha \in A\right\} .
$$


Note that $K$ is relatively compact (and hence is totally bounded). Therefore there exists a finite set $Q \subset B$ such that for any $x \in K,\|x-\tilde{x}\|<\varepsilon$ for some $\tilde{x} \in Q$.

Now let $\phi$ be the set of functions $f$ from $[0,1]$ into $B$ such that $f(i / k) \in Q$ for $i=0,1,2, \cdots, k$ and $f$ is linear on $[(i-1) / k, i / k]$ for $i=1,2, \cdots, k$. Note that $\phi$ is a finite set. We claim that $\phi$ is a $5 \varepsilon$-net with respect to $A$. Then $A$ is totally bounded and therefore is relatively compact since $C([0,1], B)$ is complete. To show this, let $f_{\alpha} \in A$. Then $\left\|f_{\alpha}(i / k)\right\| \leqq C$ for $i=0,1,2, \cdots, k$, and there exists $g \in \phi$ such that

$$
\left\|f_{\alpha}(i / k)-g(i / k)\right\|<i=0,1,2, \cdots, k .
$$

Now let $t_{0} \in[0,1]$ be such that

$$
\left\|f_{\alpha}-g\right\|_{\infty}=\left\|f_{\alpha}\left(t_{0}\right)-g\left(t_{0}\right)\right\|,
$$

and let $i_{0}$ be such that

$$
i_{0} / k \leqq t<\left(i_{0}+1\right) / k
$$

Then $\left\|g\left(i_{0} / k\right)-g\left(t_{0}\right)\right\|<3 \varepsilon$ and

$$
\begin{gathered}
\left\|f_{\alpha}-g\right\|_{\infty} \leqq\left\|f_{\alpha}\left(t_{0}\right)-f_{\alpha}\left(i_{0} / k\right)\right\|+\left\|f_{\alpha}\left(i_{0} / k\right)-g\left(i_{0} / k\right)\right\| \\
+\left\|g\left(i_{0} / k\right)-g\left(t_{0}\right)\right\|<\varepsilon+\varepsilon+3 \varepsilon=5 \varepsilon .
\end{gathered}
$$

This completes the proof of Lemma 1 .

\section{References}

[1] Billingsley, P., Convergence of probability measures, John Wiley \& Sons, Inc., New York, 1968.

[2] Dudley, R.M. and Strassen, V., The central limit theorem and $\varepsilon$-entropy, Lecture Notes in Mathematics 89, Springer-Verlag, New York, 1969.

[3] Fernique, A., Intégralité der vecteurs Gaussiens, C.R. Acaa. Sci. Paris, 270 (1970), 1698-1699.

[4] Gross, L., Abstract Wiener spaces, Proc. of the 5th Berkeley Symp. on Math. Stat. and Prob. Vol. II, Part 1, 31-42, 1966.

[5] Gross, L., Potential theory on Hilbert spaces, J. of Functional Analysis, 1 (1967), 123-181.

[6] Hartman, P. and Wintner, A.. On the law of the iterated logarithm, Amer. J. Math. 63 (1941), 169-176.

[7] JAIN, N.C., An example concerning CLT and LIL in Banach space. The Annals of Prob. 4 (1976), 690-694.

[8] Kuelbs, J., The invariance principle for Banach space valued random variables, J. of Multi. Analysis, 3 (1973), 161-172.

[9] KuELBs, J., An inequality for the distribution of a sum of certain Banach space valued random variables, Studia Math. T. LII (1974), 69-87.

[10] Kuelbs, J., A counterexample for Banach space valued random variables, The Annals of Prob., 4 (1976), 684-689.

[11] Kuelbs, J., A strong convergence theorem for Banach space valued random variables, The Annals of Prob., 4 (1976), 744-771.

[12] KuelBs, J., The law of the iterated logarithm in $C[0,1], Z$. Wahrscheinlichkeitstheorie und Verw. Gebiete, 33 (1976), 221-245. 
[13] Kuelbs, J. and LE PAGE, R., The law of the iterated logarithm for Brownian motion in a Banach space, Trans. AMS, 185 (1973), 253-264.

[14] Le PAGE, R., Log log law for Gaussian processes, Z. Wahrscheinlichkeitstheorie und Verw. Gebiete, 25 (1973), 103-108.

[15] Strassen, V., An invariance principle for the law of the iterated logarithm, Z. Wahrscheinlichkeitstheorie und Verw. Gebiete, 3 (1964), 211-226.

[16] Strassex, V., A converse to the law of the iterated logarithm, Z. Wahrscheinlichkeitstheorie und Verw. Gebiete, 4 (1966), 265-268. 